\title{
Tactile Sensors Based on Conductive Polymers
}

\author{
Julián Castellanos-Ramos ${ }^{\mathrm{a}}$, Rafael Navas-González ${ }^{\mathrm{a}}$, Haritz Macicior ${ }^{\mathrm{b}}$, Tomasz \\ Sikora $^{\mathrm{b}}$, Estíbalitz Ochoteco ${ }^{\mathrm{b}}$ and Fernando Vidal-Verdú ${ }^{\mathrm{a}}$ \\ ${ }^{a}$ Dept. of Electronics, University of Malaga, Málaga, SPAIN, vidal@ctima.uma.es \\ ${ }^{b}$ CIDETEC, Center for Electrochemical Technologies, $P^{o}$ Miramón, 196, E-20009 \\ Donostia-San Sebastián, Spain;
}

\begin{abstract}
This paper presents results from a selection of tactile sensors that have been designed and fabricated. These sensors are based on a common approach that consists in placing a sheet of piezoresistive material on the top of a set of electrodes. We use a thin film of conductive polymer as the piezoresistive material. Specifically, a conductive water-based ink of this polymer is deposited by spin coating on a flexible plastic sheet, giving it a smooth, homogeneous and conducting thin film. The main interest in this procedure is that it is cheap and it allows the fabrication of flexible and low cost tactile sensors. In this work we present results from sensors made using two technologies. Firstly, we have used a flexible Printed Circuit Board (PCB) technology to fabricate the set of electrodes and addressing tracks. The result is a simple, flexible tactile sensor. In addition to these sensors on PCB, we have proposed, designed and fabricated sensors with screen printing technology. In this case, the set of electrodes and addressing tracks are made by printing an ink based on silver nanoparticles. The intense characterization provides us insights into the design of these tactile sensors.
\end{abstract}

Keywords: Tactile sensors based on conductive polymers, sensors fabricated with printed circuit board technology, sensors fabricated with screen-printing technology.

\section{INTRODUCTION}

Tactile sensors are basically arrays of force sensors that enable monitoring across a whole specific surface area, and not only discrete points pressure monitoring. They are demanded in applications where unstructured environments or uncertainty are present, like minimal invasive surgery (MIS), robotics, rehabilitation, virtual reality, telepresence, or industrial automation (Lee 2000; Tegin and Wikander 2005). Many different approaches have been proposed to 
fabricate these sensors, most of them are based on piezoresistive (Engel et al. 2006; Kane et al. 2000; Mei et al. 2000; Lomas et al. 2004; Kim et al. 2006; Wisitsoraat et al. 2007; Shan et al. 2005) or capacitive (Salo et al. 2003; Leineweber et al. 2000; Paschen et al. 1998; Gray and Fearing 1996; Lee et al. 2006; http://pressureprofile.com/products-robotouch) principles, and a few are based on optical (Hellard and Russell 2006) or piezoelectrical transduction (Dahiya et al. 2007). Most of these sensors are made using technologies for Micro-ElectroMechanical Systems (MEMS) on silicon (Kane et al. 2000; Lomas et al. 2004; Salo et al. 2003) or on polymers (Engel et al. 2006; Kim et al. 2006; Lee et al. 2006). These technologies are not orientated to large area devices, and many of them are proposed for applications that demand high spatial resolution and good performance in terms of errors, like MIS (Salo et al. 2003). Moreover, most of these realizations have ranges in the order of hundreds of $\mathrm{mN}$ or even $\mu \mathrm{N}$. Especifications for the sensors to be used in robotics depends on the especific application (for instance fine manipulation or assistance in moving people). However, ranges in the order of tens of Newtons per tactel are common (http://pressureprofile.com/products-robotouch; http://www.shadowrobot.com/). Sensors that cover larger areas and have also wider input ranges can be obtained by arranging single force sensors on a -usually- flexible substrate (Mei et al. 2000; Shan et al. 2005; Paschen et al. 1998), but this increases costs because many instances of these force sensors are needed and because they must be assembled on the substrate. To lower the cost of the sensor it is better to obtain the whole array in the batch fabrication process minimizing further assembly of separate components. The commercial capacitive sensor of (http://pressureprofile.com/products-robotouch) is designed for manipulative tasks in robotics and is able to cover large areas and it is stretchable, so it can be mounted on free-form surfaces. However signal conditioning of capacitive sensors is more complex than that for piezoresistive sensors and most proposals for large area low cost sensors are based on piezoresistive materials, especially to obtain smart tactile sensors with very compact electronics that can be placed close to the raw sensor (Weiss and Wörn 2004; Cannata and Maggliali 2006; Shimojo et al. 2004).

A new generation of large tactile sensors are being developed and comercialized, where a piezoresistive polymer film is used (www.tekscan.com; 
http://www.peratech.com/), showing a decrease in electrical resistance when a normal force is applied. However, their use for large surface applications seems unlikely due to the high cost of materials used. The electroactive materials incorporated in currently developed configurations use metallic microparticles (silver) embedded on a polymer film, metallic central films (silver) separated by a pressurable elastomeric polymer film, or a combination of both of them (www.tekscan.com; http://www.peratech.com/). In spite of the valuable advances that these technologies show, the use of high cost materials such as silver is an important impediment since large surface applications cannot be afforded by these types of sensors. Another large area, flexible pressure sensor was proposed by Someya (Someya et al. 2004). In this case, a pressure-sensitive flexible layer made of polydimethylsiloxane (PDMS) containing electrically conductive graphite particles was used. But once again, the fabrication process was complicated as well as the cost of the final device increased by the deposition of gold layers (vacuum evaporated) and polyimide layers (cured at $180^{\circ} \mathrm{C}$ ).

The technology developed by CIDETEC (Ochoteco et al. 2008) uses conducting polymers as electroactive materials for construction of pressure sensors. The spincoating of flexible plastic films of polyethyleneterephtalate (PET) with conductive inks obtained from conductive polymers results in a flexible conductive film on flexible plastic substrates. The presence of a roughness at microscopic level leads to a resistivity decrease when different pressures are applied. This new technology shows great advantages for application on high surface area. (a) Sensor devices are light. (b) Sensors are low cost, it is thus a competitive technology to be incorporated on large surface area. (c) Flexibility in final sensor devices makes its incorporation in a flexible surface feasible.

We are interested in developing tactile sensors based on this technology. Low resolution sensors, in the range of centimeters have been successfully developed and reported (Ochoteco et al. 2008). Now we present results of sensors that are designed to be used in robotics. Specifically we focus on sensor that can be mounted on artificial hands. Spatial resolutions in the order of a few millimeters and areas in the order of square centimeters are required in this application to cover the fingers and the palm. This paper presents results from these tactile sensors. Some of them are made in a simple way. They are a set of electrodes made with Printed Circuit Board technology on a flexible substrate, with the 
plastic sheet covered with the electroactive polymer atop. A similar procedure has been followed in the sensors for robotics hands in (Weiss and Wörn 2004; Cannata and Maggliali 2006). These sensors present poor performance in terms of accuracy, hysteresis and drift, but they seem to be suitable for many applications in robotics. Moreover, local electronics can perform tasks such as compensation or smart calibration to improve the performance of the sensor. A few sensors have been fabricated in this way, with different resolutions and different geometries of the electrodes. Another set of sensors was fabricated with a screen printing technology. In this case the electrodes were printed on the substrate. An elastomer is added to confer certain benefits such as lower hysteresis and range tunability. It is also used as an insulator between conductive layers.

Several tests have been carried out to show the performance of the sensors in terms of linearity, hysteresis, dynamic response and dispersion of readings from different tactels (force sensing units in the array). The latter is not usually reported by other authors, who provide results from a single tactel. We have exerted here a uniform pressure on the surface of the whole tactile array and present results of the mean as well as the standard deviation. Results of drift are also given for different forces and when force is increased or decreased, which is not usual either. A custom pneumatic set-up was designed to also obtain measurements of the response to a pressure pulse. These tests were done on different sensors in terms of area, geometry, spatial resolution or technology. The results help us to learn more about the behaviour of the sensors and about important design issues. This will allow us to get better raw sensors and serve as a guide in the design of the electronics and algorithms to process the tactile data.

\section{FABRICATED POLYMER BASED TACTILE SENSORS}

A conducting, polymer dispersion was used as conducting ink to be applied on a flexible plastic sheet. Poly (3,4-ethylenedioxythiophene) (PEDOT) was chosen as the conducting raw material, and aqueous dispersions of this polymer were prepared for the elaboration of conducting inks. PEDOT aqueous dispersions were prepared by using an Ultrasonic Processor (model UP $400 \mathrm{~S}$ from Dr. Hielscher $\mathrm{GmbH})$ during the synthesis. As an example, ethylenedioxythiophene (1 ml, 9.4 
$\mathrm{mmol}$ ) and $3.5 \mathrm{~g}$ of poly styrene sulfonate (PSS) were dissolved in $100 \mathrm{ml}$ of distilled water. To this mixture, an equimolar amount of ammonium peroxydisulphate $(6.58 \mathrm{~g}, 28.8 \mathrm{mmol})$ dissolved in $50 \mathrm{ml}$ of water was added dropwise over a period of $4 \mathrm{~min}$. After $1 \mathrm{~h}$ of reaction under ultrasonic irradiation a dark blue PEDOT aqueous dispersion was obtained.
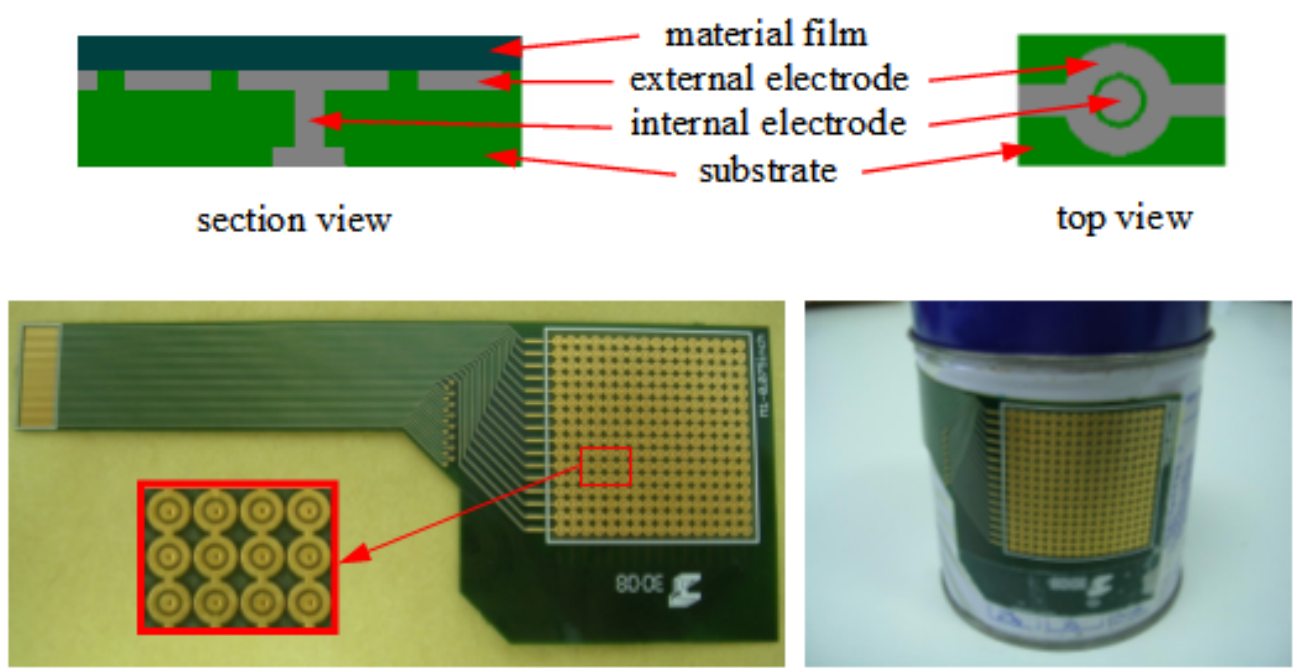

Fig. 1. Tactile sensor based on a flexible Printed Circuit Board

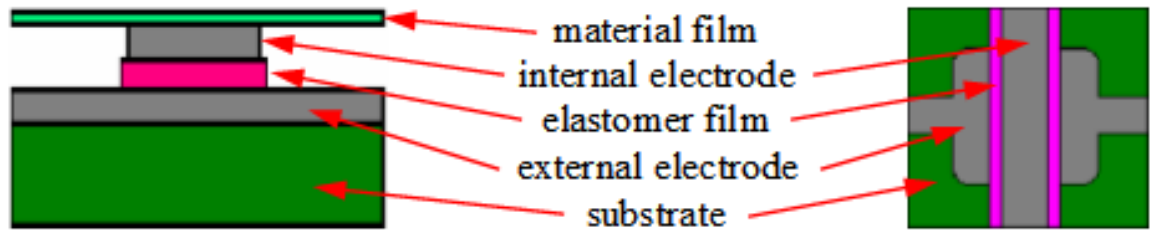

section view top view

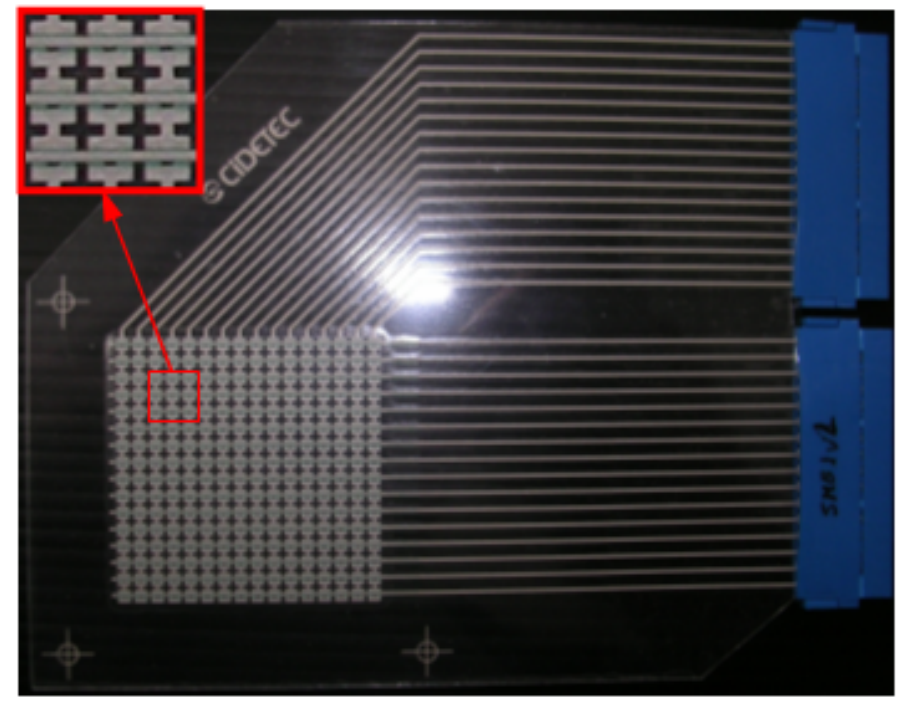

Fig. 2. Tactile sensor made with a screen printing technology. 
Conducting flexible sheets were prepared placing a PET (polyethyleneterephtalate, $5 \times 5 \mathrm{~cm} 2$ in surface, $70 \mathrm{~m}$ in thickness) plastic sheet on a spin coater. Fifty microliter of conducting polymer ink were dispensed on it and it was rotated at 1,500 rpm. After $1 \mathrm{~min}$, it was stopped and left to dry at room temperature. A thin conducting film was created on the plastic sheet, giving a conducting flexible plastic sheet.

A key issue of the obtained conductive plastic film is the microscopic roughness with an average grain size of $50 \mathrm{~nm}$ because the working principle of the sensor is the variation of the effective area of contact between the polymer and the electrodes at microscopic level and the change of the conductance as a consequence. These electrodes can be fabricated with a Printed Circuit Board technology. Fig. 1 shows a section view and a top view of a obtained tactel as well as photographs of a sensor fabricated in this way. The electrodes are $\mathrm{Cu} 18 \mathrm{um}$ thick chemical gold plated and the substrate is polyimida $0.2 \mathrm{~mm}$ thick. The sensors of this paper have been fabricated with this technology. Fig. 2 shows an example of implementation of a sensor with screen printing technology. Again, a section view and a top view are depicted. Note that an elastomer is placed between the two tracks to implement insulation and prevent the tactel to be short circuited.

\section{MEASUREMENT SET UP}

The measurement set-up we have used to characterize the sensors consists of devices to exert force or pressure against the surface of the tactile sensors and signal conditioning electronics to read the information from the sensors. Many tests were made with a set-up that consisted of a translation stage to place the sensor on, a stepper motor to exert the force via a spring and a force sensor placed at the tip of the probe (Castellanos et al. 2009). Similar systems are described in other reported works. An important question arises if we use the previous, common set-up to compare different sensors. It is the mismatching between tactels, which is quite important in this kind of sensor. Since our purpose is to find some insights into the behaviour and design issues of these sensors, we have to test and compare different designs. Therefore, to obtain significant conclusions, 
we should characterize not only a tactel but the tactile sensor as a whole. Then we compare the mean value of the measurements provided by all the tactels of a sensor with others obtained from the set of tactile sensors under study. This will allow us to see the effects of different design decisions without being masked by mismatching between tactels.

To exert a uniform pressure on the sensor we have used the pneumatic device in Fig. 3. It is commercialized by Tekscan (www.tekscan.com) to be used to equilibrate their sensors (see section 4.6) Equilibration is a procedure recommended to compensate mismatching between tactels. We will use this device to characterize our sensors in the way described. The device has a pressurized chamber with a flexible wall or membrane. This wall is in close contact with the sensor and almost does not bend, so the pressure on the tactels is practically the same. The pressure in the chamber is set by a knob and its value is displayed by an analogue manometer. We added a second digital manometer to improve the set-up to be used to characterize the sensor and not just to equilibrate it.
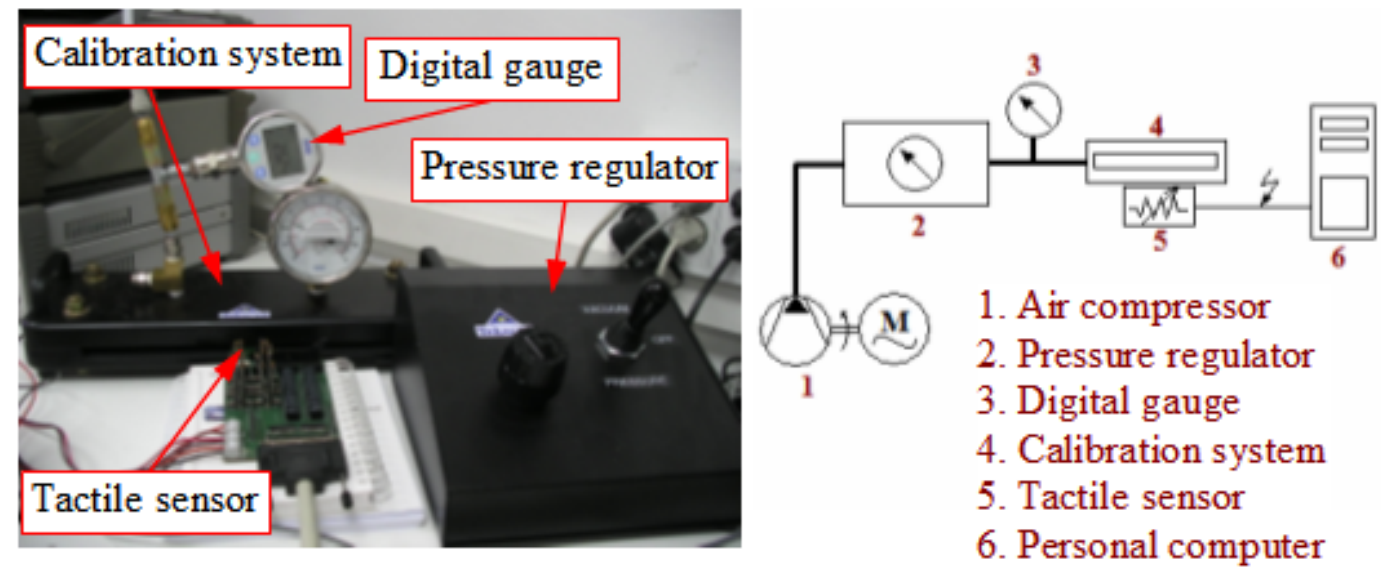

Fig. 3. Set up to measure the static response of the sensor. 


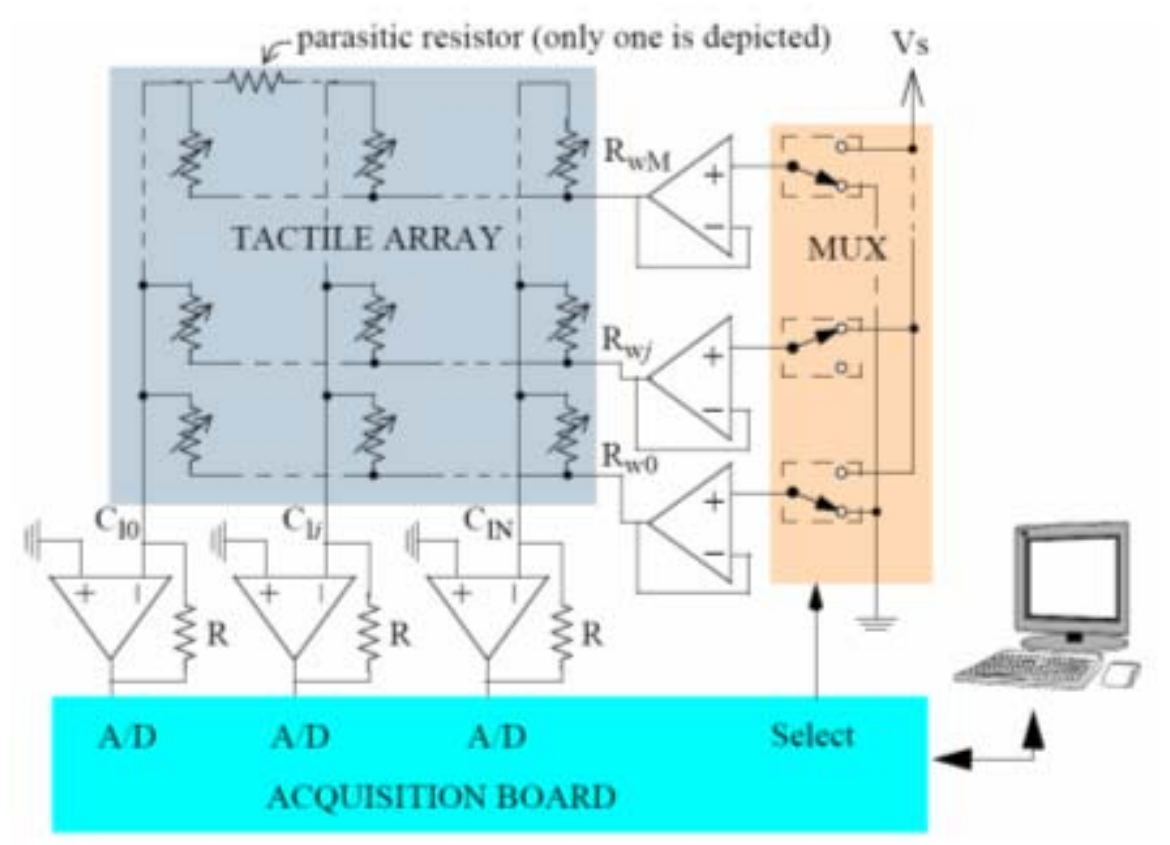

Fig. 4. Electronics developed to read the data from the raw tactile sensors.

Regarding the electronics, Fig. 4 shows a common mode of implementation. The raw sensor can be modelled by an array of resistors that correspond to those between the inner and outer electrodes at Fig. 1. However, other lateral resistors are also present in the real sensor and can be modelled as resistors between the electrodes of different tactels in the arrays of Fig. 4. Even if these resistors would not exist, i.e we had a discrete set of force sensors as tactels, crosstalk is present as long as tracks are shared by them in order to be addressed in rows and columns. When the array is addressed in this way parasitic resistive paths arise. This source of error is masked by the crosstalk caused by parasitic paths in continuous piezoresistive films and is easily underestimated. The circuitry in Fig. 4 reduces the undesired contribution of crosstalk to the reading (D'Alessio 1999). This is because the output amplifier forces all output nodes to be virtually grounded. Since the rows that are not driven are also grounded, parasitic resistors are virtually short-circuited and do not contribute to the output. The results in the next section were obtained using this circuitry. The array is read sequentially and only one row is driven at a time. The output of this circuit was read by an acquisition board in a PC.

Besides the static characterization, some tests were made to better understand the dynamic behaviour of the sensors. Firstly, we would like to know the response to 
a sudden increase and decrease of the pressure on a tactel, so we had to build a device to create a pressure pulse on the sensor surface. The device used for the static characterization did not provide a fast enough response, so we had to design and mount a specific test bed, as is shown in Fig. 5. The chamber is that of the device described in the above paragraph, so that device allows us to obtain a given target pressure of the pulse. To generate such a pulse a three way electrovalve is set to connect the chamber with a short pipe ended with a flexible membrane in close contact with the sensor surface. The pressure increases suddenly up to the value set by the regulator. To end the pulse the valve is set to connect the pipe to the atmosphere, so the pressure inside falls quickly.

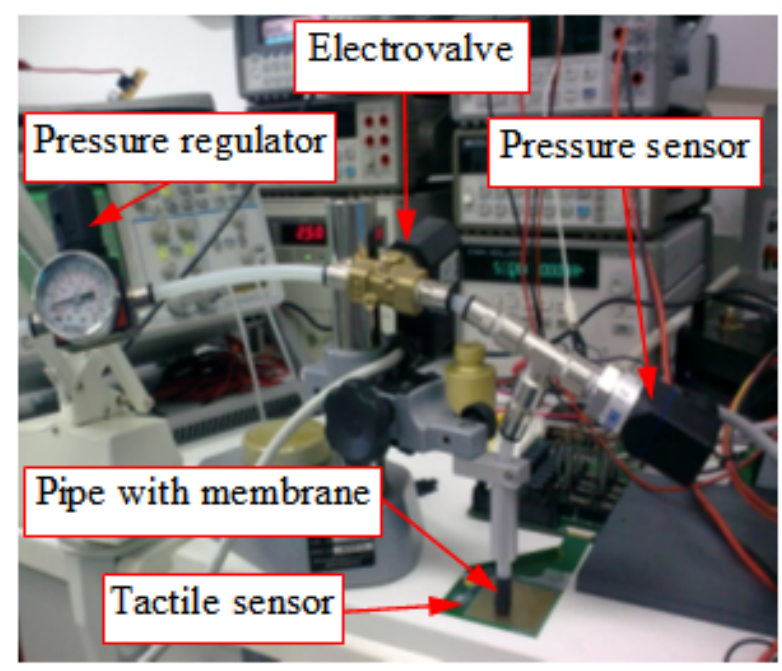

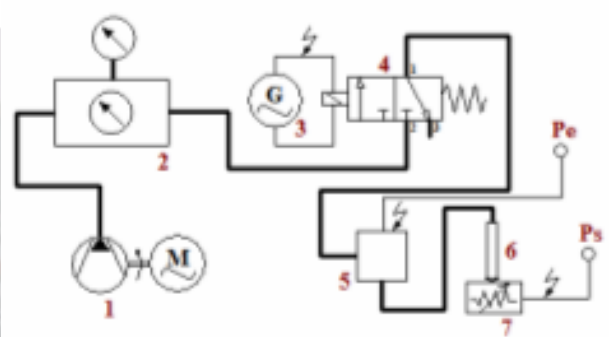

1. Air compressor

2. Pressure regulator with manometer

3. Driving circuit for the electrovalve

4. Three ways electrovalve

5. Pressure sensor

6. Pipe with membrane

7. Tactile sensor

Pe. Input test point

Ps. Output test point

Fig. 5. Set up developed to measure the dynamic response of the tactile sensors.

\section{RESULTS AND DISCUSSION}

Many tests were made with the measurement set-up and the fabricated sensors that have been shown in the previous sections. Here we will show some results and discussions that will include comparisons to illustrate the main features of the sensors and their design. Sensors on Printed Circuit Board are made on $200 \mu \mathrm{m}$ thick Polyimida with gold electroplated $18 \mu \mathrm{m} \mathrm{Cu}$ and the $70 \mu \mathrm{m}$ thick $0.082 \mathrm{~S} / \mathrm{cm}$ conductivity piezoresistive polymer sheet atop, unless otherwise noted. 


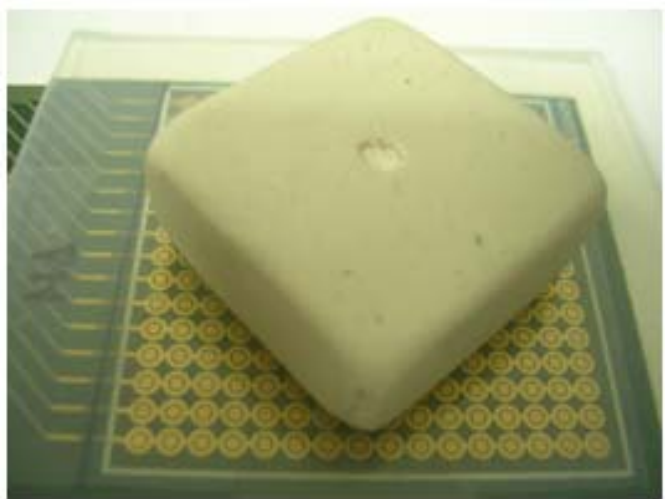

(a)

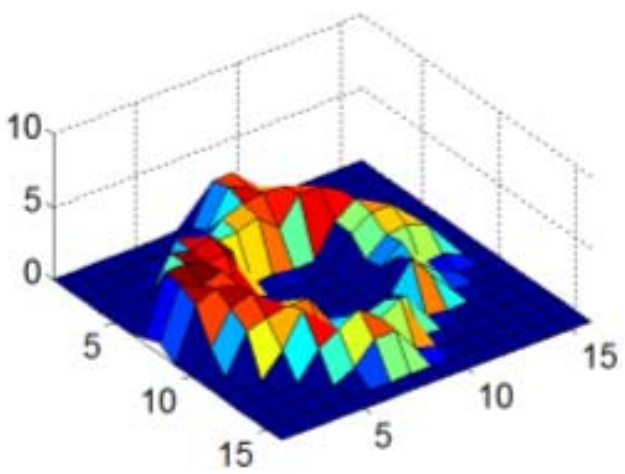

(b)

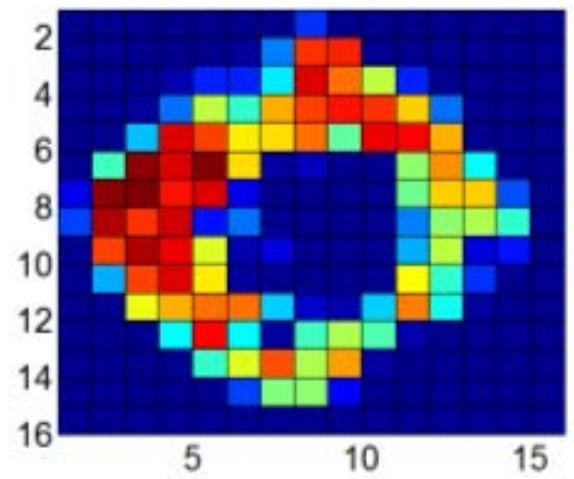

(c)

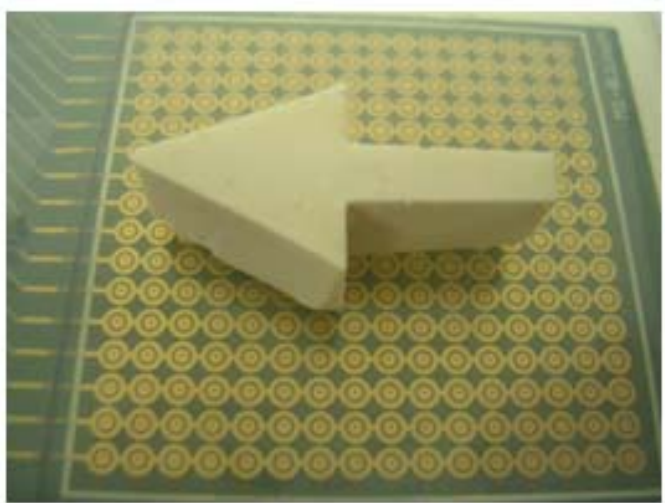

(d)

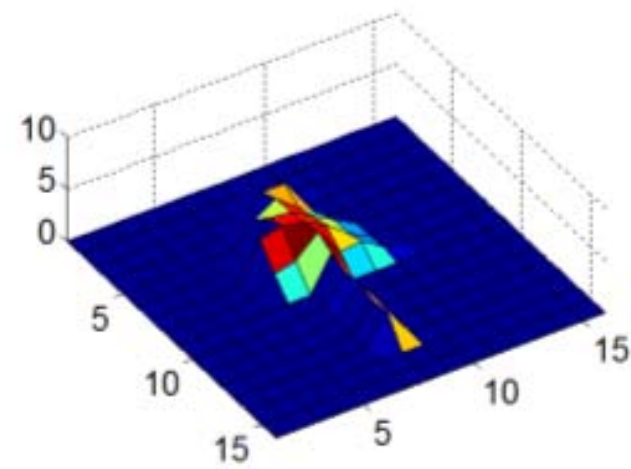

(e)

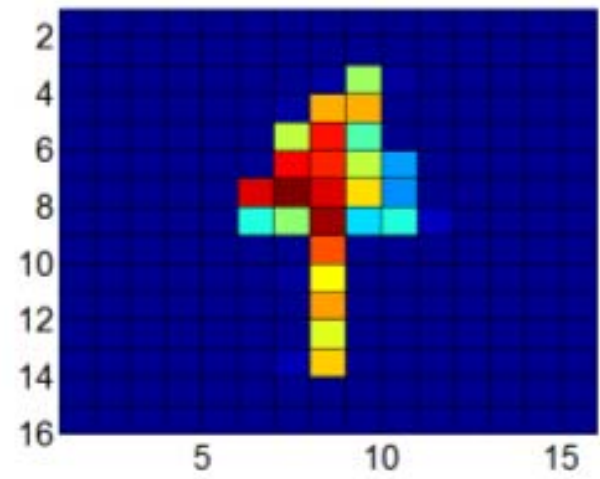

(f)

Fig. 6. 2D and 3D output of one sensor when a rubber with a hole (a)(b)(c) or an arrow made of rubber $(d)(e)(f)$ are pressed against its surface.

Besides the results of the dynamic behaviour, the static performance of the sensors is illustrated by measurements made using the set-up shown in Fig. 3. For instance, Fig. 8 (a) shows results from five cycles (every cycle takes 4 minutes approximately) where the pressure was increased and then decreased. The curves at the top of the figure show the mean value of the readings of all the tactels in the 
array (only a few outliers beyond twice the standard deviation were removed), while the curves at the bottom show the standard deviation of the reading with respect to the mean value. A quite large mismatching between tactels is observed. We will comment on this later together with some strategy to reduce it. Fig. 8 (b) shows the mean value of the data from previous figure, i.e. the curves with the mean value of the readings of all tactels are also promediated and the result is displayed together with its standard deviation. Note that the standard deviation is quite low here, so the measurements are quite repeatable. Finally, Fig. 6 shows the tactile image obtained by the system from a simple test that consists in pressing the sensor surface with a rubber. A hole has been made in the center of the rubber in the case of Fig. 6 (a). Axis $z$ in Fig. 6 (b) represents the force exerted and $x$ and $y$ axis correspond to the coordinates of the tactels in the sensor. A top view of this image is shown in Fig. 6 (c). A similar experiment was made with an arrow made of rubber Fig. 6 (d), and the results are shown at Fig. 6 (e) and Fig. 6 (f).

In the following, some interesting issues will be discussed related to sensors on PCB technology whose data is at Table 1 and others made on screen-printing: area of the electrodes, geometry of the electrodes, conductivity of the polymer film, dynamic response and sensors made with screen printing technology.

Table 1. Shape and size of electrodes made with PCB technology.

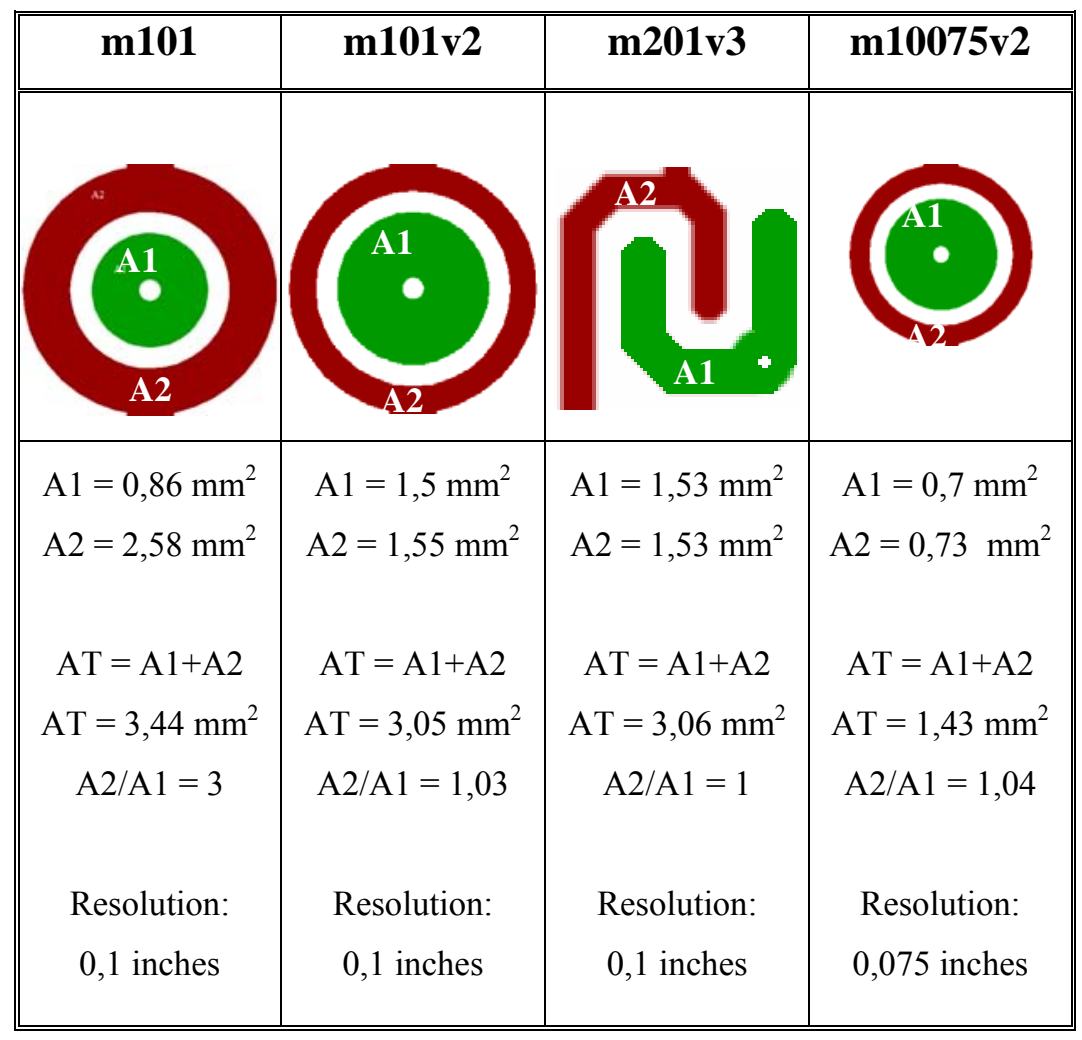




\subsection{Area of the electrodes}

In order to see the influence of the area of the electrodes on the sensor performance we fabricated a few arrays on PCB. A first significant result was that electrodes had to be designed in such a way that the areas of both were the same. Otherwise the performance of the sensor is determined mainly by the area of the smaller one. The simple model in Fig. 7 can illustrate this.

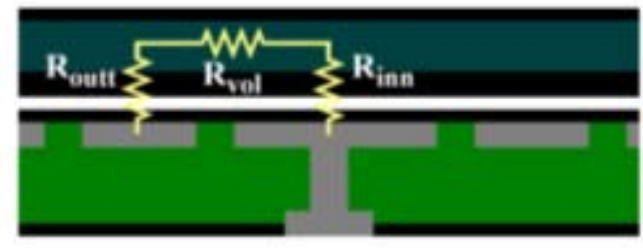

Fig. 7. Simple model of the resistive path between the electrodes of a tactel.

The equivalent resistance of a tactel is given by the equation

$R_{\text {eq }}=R_{\text {inn }}+R_{\text {vol }}+R_{\text {out }}$

Where $R_{\text {inn }}$ and $R_{\text {out }}$ are the resistances at the interfaces with the inner and outer electrodes respectively and $R_{v o l}$ is the resistance of the path between both electrodes. As mentioned in section 2, the resistance depends on the effective contact area that depends also on the pressure and is obviously proportional to the area of the electrode. Therefore we can write

$R_{\text {inn }}=\frac{a(P)}{A_{\text {inn }}} \quad R_{\text {outt }}=\frac{a(P)}{A_{\text {outt }}}$

Where $A_{\text {inn }}$ and $A_{\text {outt }}$ are the area of the inner and outer electrodes respectively and $a(P)$ is a function of the pressure exerted on the sensor. It is obvious that the summation of both areas is a constant for a given spatial resolution of the sensor, so $A_{\text {inn }}+A_{\text {outt }}=A$, where $A$ is a constant. Therefore, for a given pressure on the sensor it is readily obtained from (2) that

$\frac{1}{R_{\text {inn }}}+\frac{1}{R_{\text {outt }}}=C$ 
Where $C$ is a constant. From (1) and (3) it is obtained that $R_{e q}$ has a minimum for $R_{\text {inn }}=R_{\text {outt }}$, and from (2) it is concluded that $A_{\text {inn }}=A_{\text {outt }}$.

Fig. 8 shows measurements that confirm this conclusion. The output at Fig. 8 (a) and (b) corresponds to the sensor with the data shown at Table 1 in the column labelled with m101. Fig. 8 (c) and (d) show the output for the sensor with label m101v2. Note that even with a smaller total area (summation of the area of both electrodes) the sensitivity of the sensor with balanced electrodes is larger. In addition, Fig. 9 shows the influence of the area or resolution. This figure shows the output of the sensor labelled with m10075v2 at Table 1. For smaller areas $R_{\text {inn }}$ and $R_{\text {outt }}$ increase (see eq. (2)) and the sensitivity of the sensor is reduced.

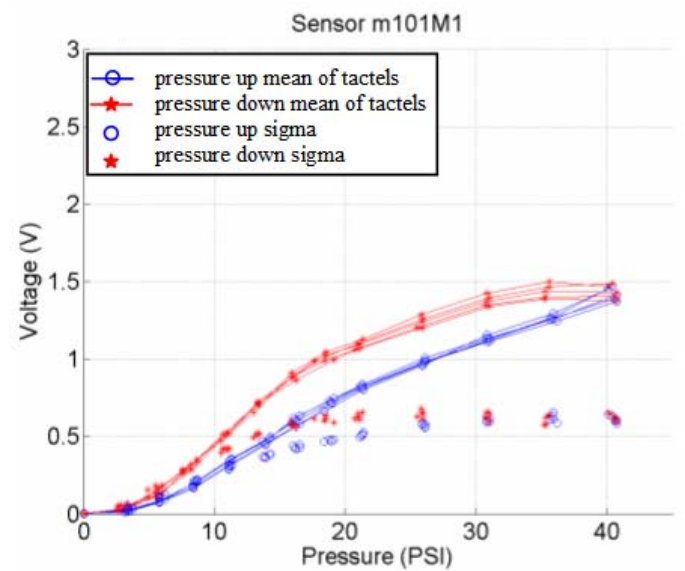

(a)

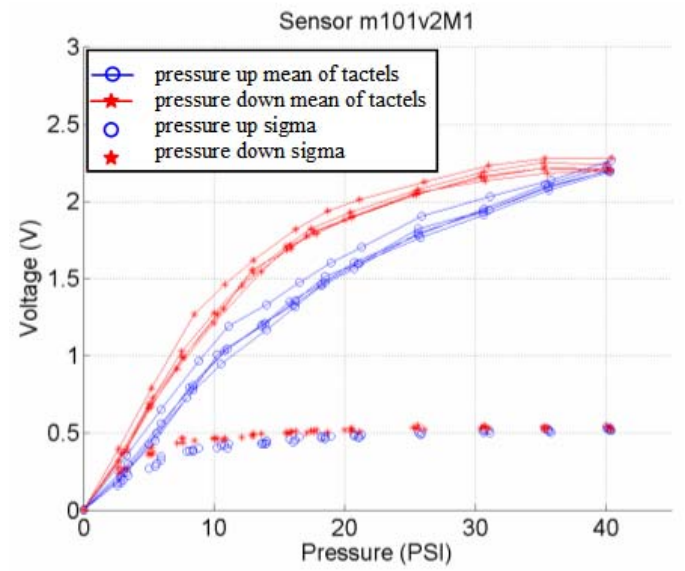

(c)

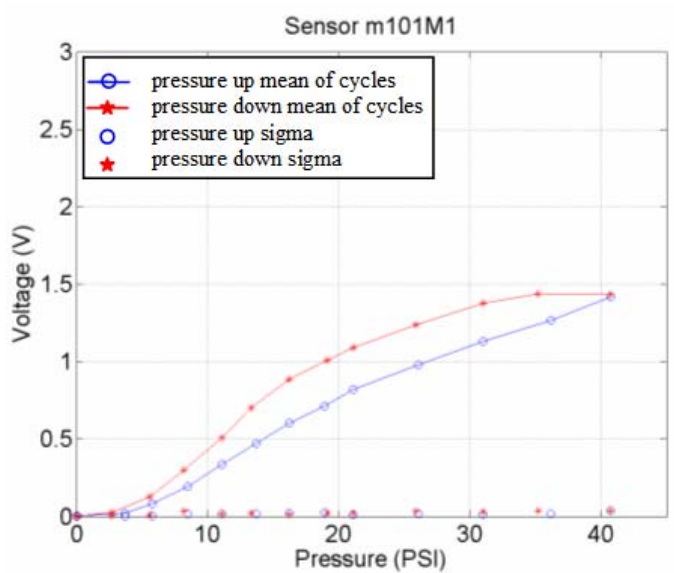

(b)

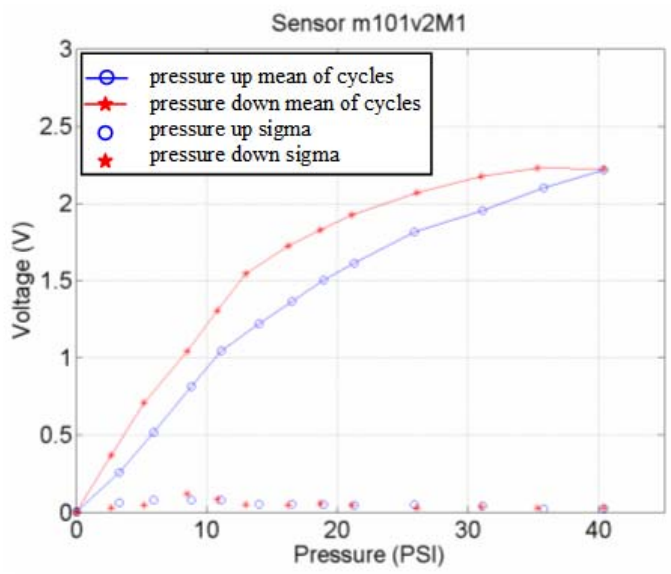

(d)

Fig. 8. Mean and standard deviation of the output from all tactels for five measurement cycles from the sensor $\mathrm{m} 101$ in Table 1 (a) and average and deviation of these cycles (b) and the same for sensor m101v2 in Table 1 (c) and (d). 

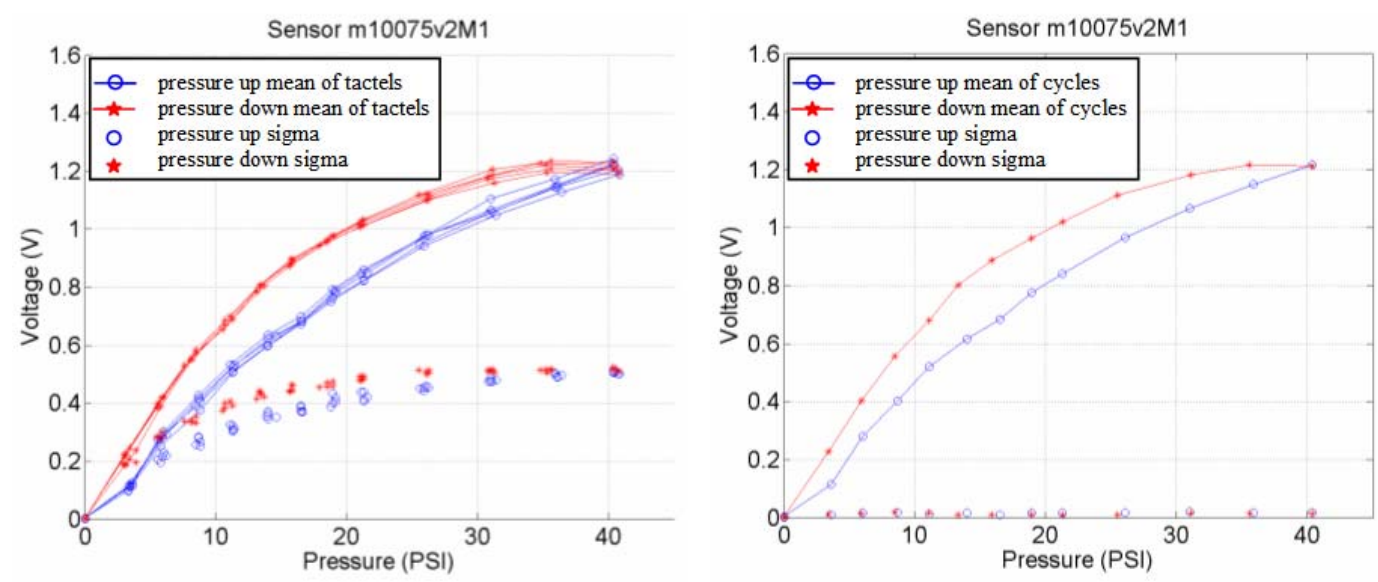

Fig. 9. Output from a high resolution sensor (m10075v2 in Table 1$)$.

\subsection{Geometry of the electrodes}

We also wanted to know the influence of the shape of the electrodes on the behaviour of the sensor. We made another array of electrodes on the same PCB technology. The area of each electrode was the same as that of the sensor whose output is at Fig. 8 (c) and (d), but their shape was a kind of comb, which is also quite common (Ochoteco et al. 2008). Therefore we compare sensors with the electrodes of the same area but different geometries, circular and comb shaped. The results of the latter are shown at Fig. 10 . Besides slight differences in the shape of the curve and the sensitivity, two main differences can be highlighted. First, a larger hysteresis in the sensor with the comb electrodes can be observed. Second, a larger mismatching between tactels is also observed in this case.
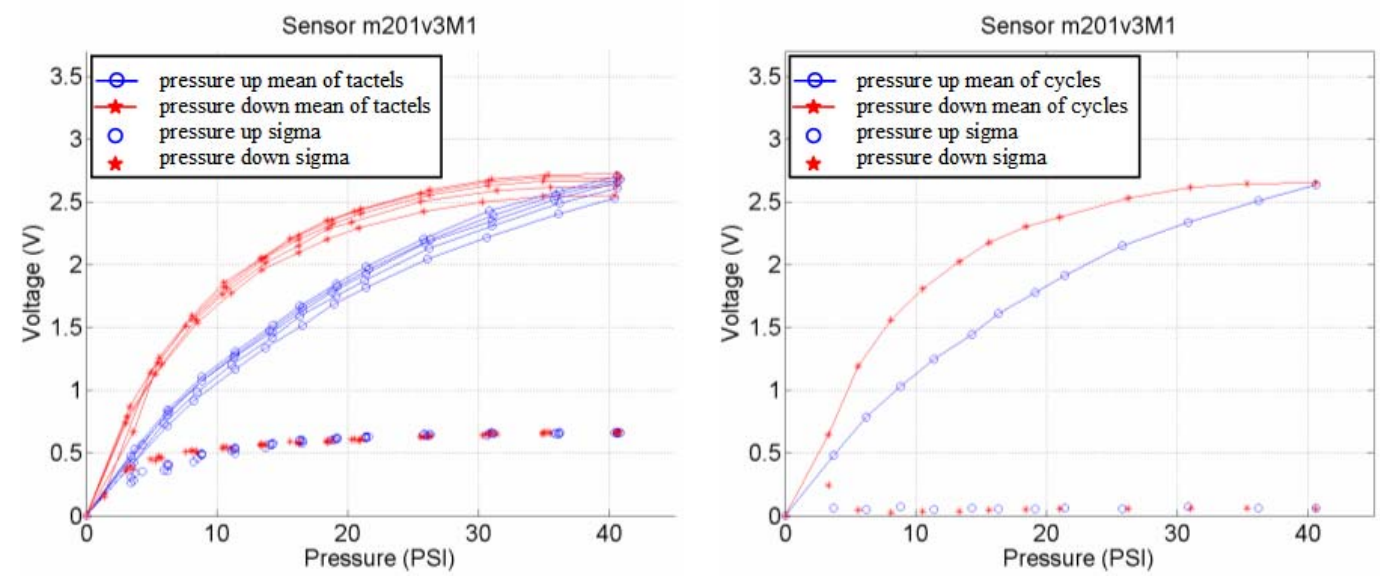

Fig. 10. Output from a sensor with comb shaped electrodes (m201v3 in Table 1). 


\subsection{Conductivity of the polymer film}

Based on the idea that the adequate control of electrical conductivity values and film morphologies allow working pressure ranges to be tailor-made designed, a synthesis strategy is developed, obtaining conducting polymers with a wide range of surface morphologies and electrical conductivities. Concretely, some PEDOTAu nanocomposites are designed using PSS dopant. In these cases, the electrical conductivity of the film increased compared to the electrical conductivity measured in the films in absence of $\mathrm{Au}$ nanoparticles. Moreover, the film morphology showed a clear increase in roughness in PEDOT/PSS/Au films compared to PEDOT/PSS films. A surface roughness of 50nm appears for the PEDOT/PSS film while a roughness of $75 \mathrm{~nm}$ can be observed for the PEDOT/PSS/Au one. Regarding the final sensor performance, the conductance increased when PEDOT/Au nanocomposites were used as electroactive material in the device. This can be exploited to increase the sensitivity of the sensor, for instance in the case of having a high resolution sensor. This is illustrated at Fig. 11, where the output of two sensors with geometry labelled as m10075v2 at Table 1 is displayed. The curve at the bottom is obtained with a polymer with conductivity $0.082 \mathrm{~S} / \mathrm{cm}$ while the curve at the top corresponds to a polymer with conductivity $0.169 \mathrm{~S} / \mathrm{cm}$. A clear increase in the sensitivity of the sensor can be observed.

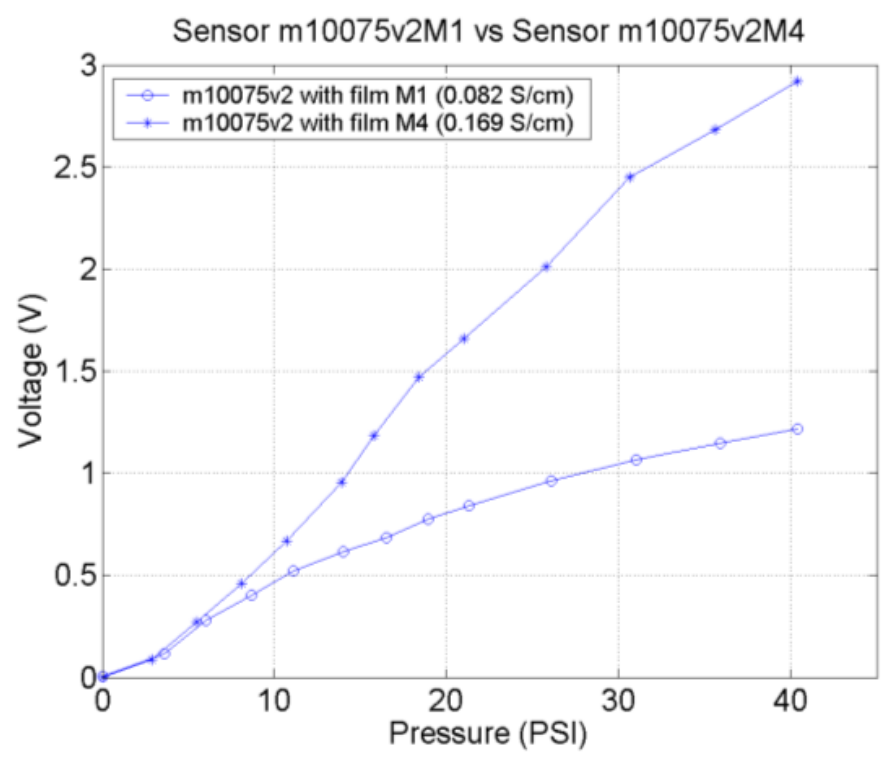

Fig. 11. Response of the high resolution sensor m10075v2 in Table 1 with polymers of different conductivity. 
For the preparation of the gold nanoparticles, a freshly prepared $10 \mathrm{ml} 5 \times 10^{-4} \mathrm{M}$ solution of $\mathrm{NaBH}_{4}$ was added dropwise under vigorous stirring to $10 \mathrm{ml}$ of a $4,2 \times 10^{-4} \mathrm{M}$ gold tetrachloroauric acid solution. The solution was further stirred for 15 minutes and pink colour dispersion was obtained. A typical UV-Visible absorption spectrum shows a Surface Plasmon peak at 524nm. Average sizes of the gold NP measured by Dynamic Light Scattering and TEM were 3nm and $3.2 \mathrm{~nm}$, respectively. This solution was added to PEDOT dispersion obtaining different final electrical conductivities

\subsection{Dynamic response}

Two tests where carried out to obtain information about the dynamic performance of the sensor. The first one consisted of exerting a pressure pulse against the surface of the sensor on a tactel. For this purpose we used the set-up shown in Fig. 5 and described in section 3. Fig. 12 (a) shows the output of the sensor (top) for a pressure pulse of amplitude $22 \mathrm{psi}$ and a resistance $\mathrm{R}=470 \mathrm{ohms}$ in the electronics of Fig. 4 and the sensor labelled m101v2 at Table 1. Enlargements of the rise and fall edges are also shown in the figure. The bottom curve in all figures is the reading provided by the pressure inside the pipe by the pressure sensor at Fig. 5 (http://www.wika.es). As can be observed in the figures, the curves from the tactile sensor follow those from the pressure sensor quite closely and we conclude that the dynamic response is in the order of a few milliseconds.

Another test was made to see the drift of the sensors. Other authors usually only provide the drift for a given pressure, commonly exerted by a dead weight placed on the sensor. We have followed a procedure similar to that reported in (Hollinger and Wanderley 2006) for a characterization of commercial force sensors based on similar principles. This procedure consists in measuring the drift not only from a point of zero pressure being applied, but also when existing pressure is incremented. We also show results of positive as well as negative increments. Fig. 12 (b) shows the results of this experiment that was performed with the set-up shown in Fig. 3 . Three significant issues are worth mentioning here. Firstly, a large drift is observed in the case that the sensor is not loaded at the starting point. This is also reported by other authors (Kerpa et al. 2003) and a preload can 
improve the behavior of the sensor (Hollinger and Marcelo 2006; Cannata and Maggliali 2006).

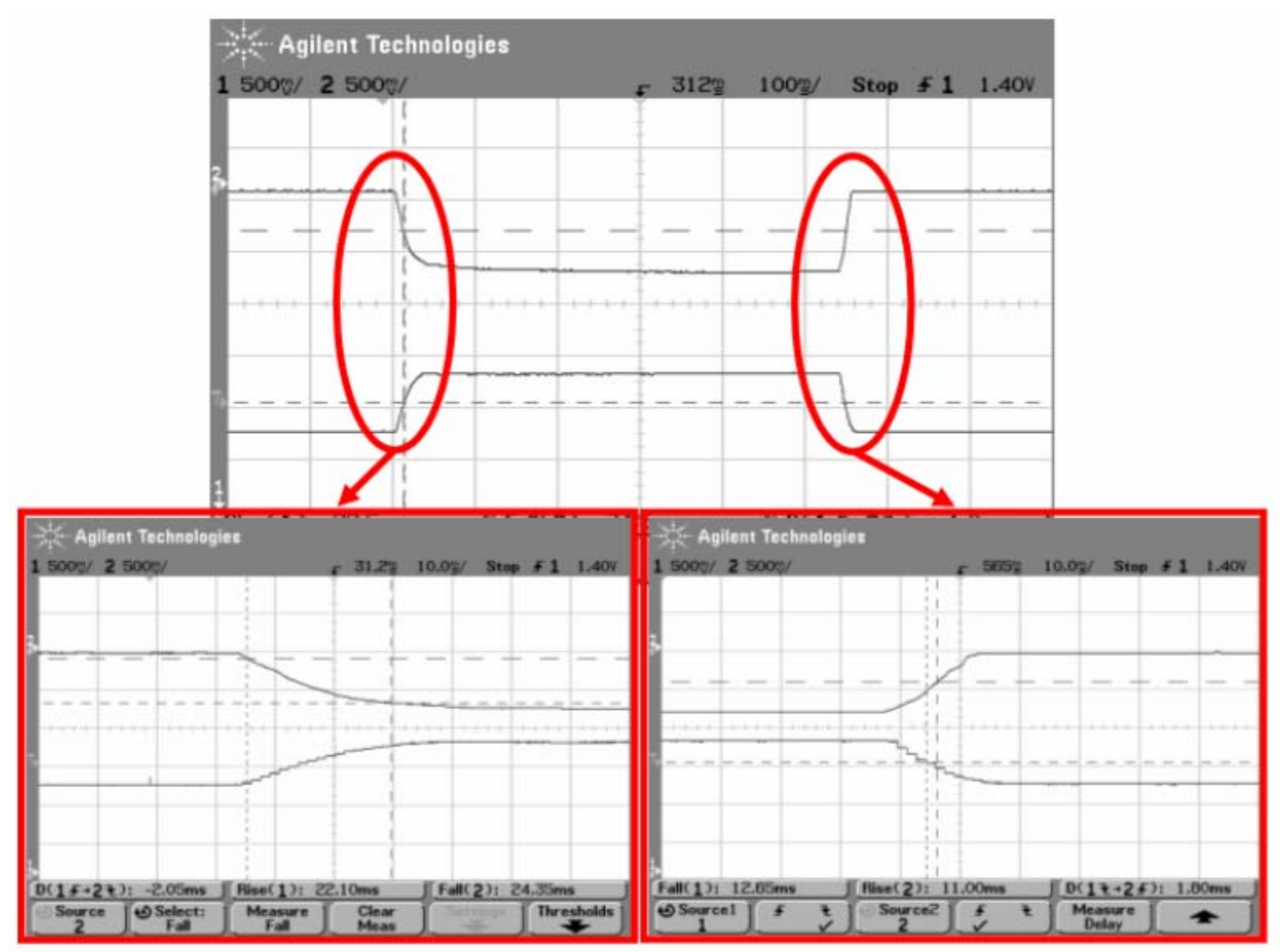

(a)

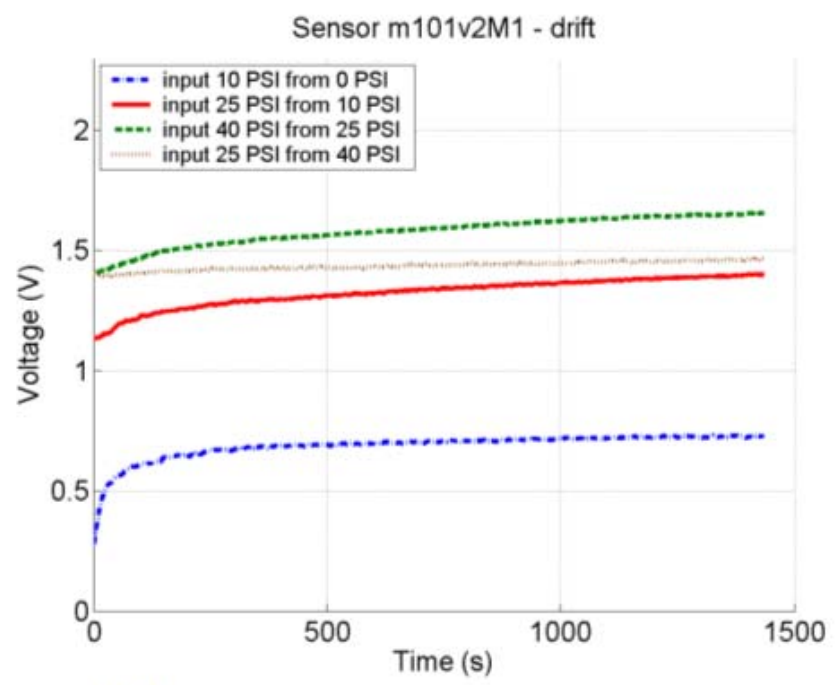

(b)

Fig. 12. Dynamic characterization of the tactile sensors based on PCB: response to a pressure pulse (a) and drift (b). 
Secondly, for the remaining curves (except that not preloaded), a drift below $15 \%$ is observed in the worst case until the end of the test that takes $1,794 \mathrm{~s}$. The values for the drift are similar to those reported in (Hollinger and Marcelo 2006) for commercial force sensing resistors. Finally, it is worth noting that the drift is very little for negative increments in the pressure.

\subsection{Sensors made with a screen-printing technology}

In addition to the sensors on PCB whose results have been shown above, similar sensors were fabricated with screen printing. These sensors are described in section 2. Fig. 13 summarizes the results obtained from the same tests described in previous sections and realized with the setups in Fig. 3 and Fig. 5 . We can see in Fig. 13 (a) and (b) that the hysteresis is lower in this sensor. On the other hand, a quite large pressure threshold can be observed. However, this threshold could be reduced by changing the compliance of the inner elastomer and also a preload can be applyied to overcome its practical effects. The presence of this threshold is also noticeable in the dynamic response to a pressure pulse shown at Fig. 13 (c) that is in the order of a few milliseconds also. For curves starting from pressures other than zero, the drift is below $16 \%$ in the worst case (see Fig. 13 (d)). The drift is also very little for negative increments in the pressure. 


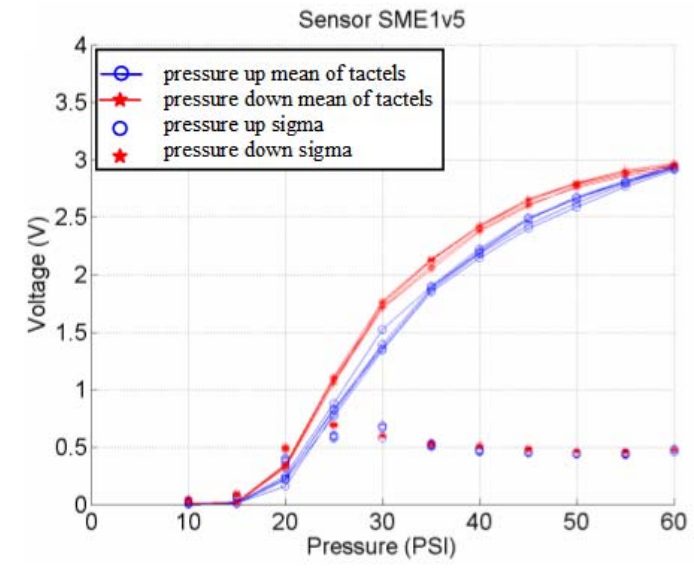

(a)

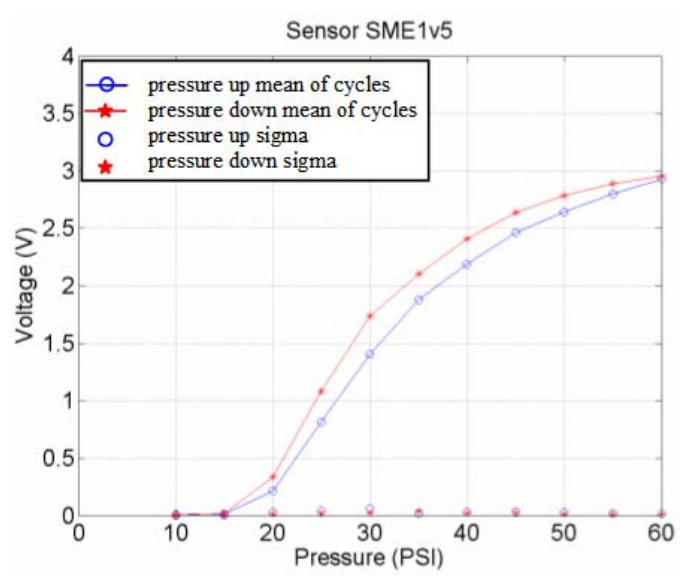

(b)

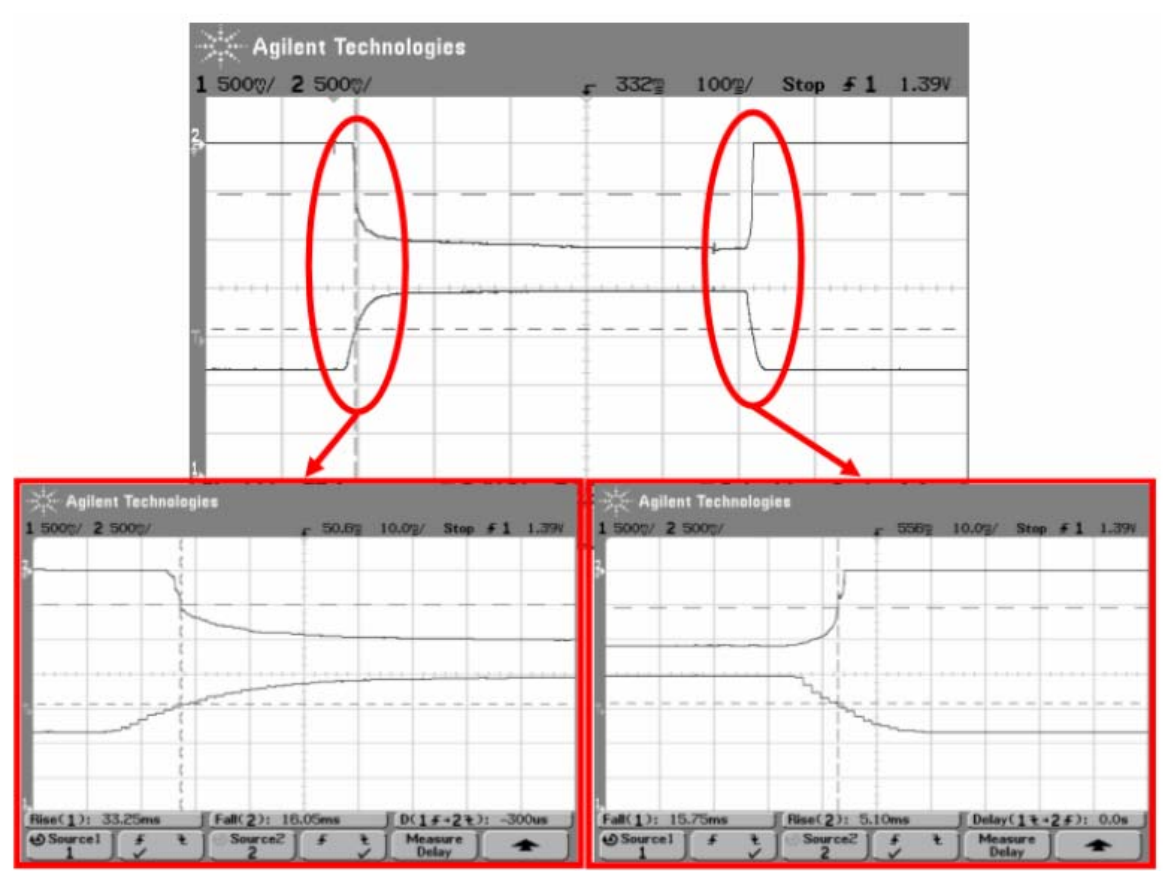

(c)

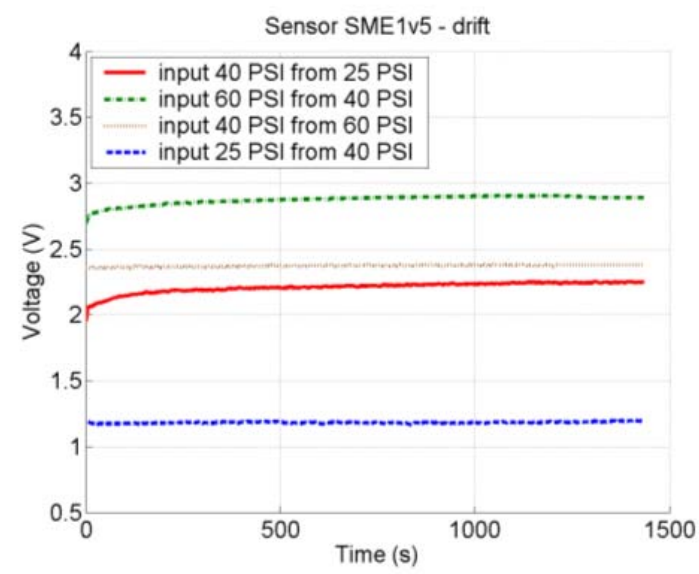

(d)

Fig. 13. Results from a tactile sensor fabricated with screen printing: curves for five cycles of increasing and decreasing pressure (a), mean (top) and standard deviation (bottom) of previous curves (b), response to a pressure pulse (c) and measurements of drift (d). 


\subsection{Further processing (equilibration)}

As can be seen in previous results, some undesired source of errors such as hysteresis, drift or mismatching are present in these sensors. This is common to sensors made with similar approaches (Weiss and Wörn 2004; Cannata and Maggliali 2006; Shimojo et al. 2004). In order to use them, some processing is required to compensate these errors. For instance, the equilibration (www.tekscan.com) of the sensor to reduce the mismatching between tactels. If $m_{i j}$ is the measurement from a tactel, the equilibration is made as

$m_{i j(e q)}=m_{i j} \times \frac{\text { mean_eq }}{m_{i j \_} e q}$

where $m_{i j \_} e q$ is the output of the tactel for a given pressure at the equilibration point and mean_eq is the mean value of the outputs from all tactels at the equilibration point (it should be the same without mismatching). Fig. 14 shows an example of the result of this procedure. The input range has been divided into three intervals where three different equilibration points (15 psi, $25 \mathrm{psi}, 35 \mathrm{psi}$ ) have been used. A lower mismatching is observed in the equilibrated sensor.

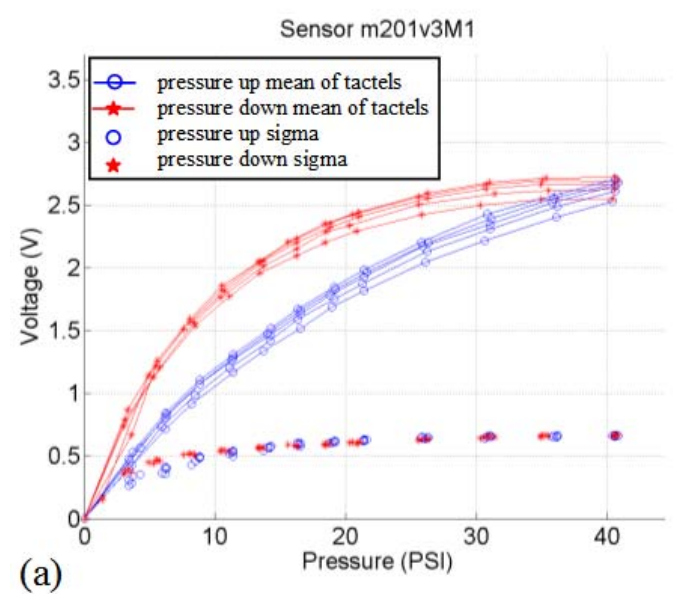

Fig. 14. Results obtained from a sensor without equilibration (a) and with equilibration (b). 


\section{CONCLUSIONS}

This paper presents results from tactile sensors based on electroactive polymers. Many tests were made on different sensors to learn more about their behaviour. Some results are shown here together with a few valuable insights on the design issues and performance. It is worth noting that we provide results obtained with a uniform pressure against the sensor. In this way we can compare different approaches that are not masked by mismatching between tactels. This mismatching is high, so at least some procedure such as equilibration, recommended by other commercial sensors, is also required here to obtain more resolution. We also conclude that the area of the electrodes in a tactel should be balanced to achieve maximum sensitivity. Improved performance in terms of hysteresis and mismatching was also observed for sensors with circular concentric electrodes when compared to sensors with comb shaped electrodes. Spatial resolutions as low as 0.05 inches between centres of tactels have been successfully tried. The sensitivity is lower in this case because of the smaller area of the electrodes, but this can be compensated for with tailor-made polymers with higher conductivity. Sensors based on flexible printed circuit boards as well as others based on screen printing were fabricated. The latter show notably reduced hysteresis. A specially developed set up was used that allowed us to carry out repeatable tests to measure the dynamic response to a pressure pulse. The results from several trials show delays in the order of a few milliseconds. Other tests show the drift for negative and positive increments of the pressure. Drifts below $16 \%$ during a period of 1,794 seconds for preload sensors are measured, which is similar to other sensors, for instance force sensing resistors based on a similar technology. We can conclude that valuable information was obtained regarding how to design these sensors and also how to use them, since undesired effects such as mismatching or drift should be taken into account in the processing of data from the raw sensor by the local electronics of a smart tactile sensor. This is the common approach to obtain low cost, relatively large area sensors for robotics. 


\section{ACKNOWLEDGEMENTS}

This work has been partially funded by the spanish government under contract TEC2006-12376-

$\mathrm{C} 02$.

\section{REFERENCES}

Cannata, G. and Maggliali, M. (2006) "Processing of Tactile/Force Measurements for a Fully Embedded Sensor", Proceedings of the 2006 IEEE International Conference on Multisensor Fusion and Integration for Intelligent Systems, pp. 160-166, September 2006.

Castellanos Ramos, J., Navas González, R., Macicior, H., Ochoteco, E. and Vidal Verdú, F. (2009) "Tactile sensors based on conductive polymers", Smart Sensors, Actuators and MEMS IV, Proceedings of SPIE Vol. 7362, 73620G-1, 2009.ISBN:9780819476364.

Dahiya, R.S., Valle, M., Metta, G. and Lorenzelli, L. (2007) "POSFET Based Tactile Sensor Arrays", 14th IEEE International Conference on Electronics, Circuits and Systems, 2007, ICECS 2007, pp. 1075-1078

D'Alessio, T. (1999) "Measurement errors in the scanning of piezoresistive sensors arrays", Sensors and Actuators, A72, pp.71-76, 1999.

Engel, J. , Chen, N., Tucker, C., Liu, C., Kim, S.-H. and Jones, D. (2006) "Flexible Multimodal Tactile Sensing System for Object Identification”, Proc. of the IEEE Sensors 2006, Exco, Daegu, Korea, October 2006, pp. 563-566.

Gray, B.L. and Fearing, R.S. (1996) “A Surface Micromachined Microtactile Sensor Array “, IEEE Int. Conf. Robotics and Automation, April 1996.

Hellard, G. and Russell, R. A. (2006) "A Tactile Sensor Array that also Grasps Objects", Proceedings of the Australasian Conference on Robotics and Automation 2006, Auckland, New Zealand ,December 6 - 8, 2006

Hollinger, A. and Wanderley, M. (2006) "Evaluation of Commercial ForceSensing Resistors", International Conference on New Interfaces for Musical Expression, NIME06, Paris 2006

Kane, B. J., Cutkosky, M. R. and Kovacs, G. T. A. (2000) "A Traction Stress Sensor Array for Use in High-Resolution Robotic Tactile Imaging", Journal of Electromechanical Systems, Vol. 9, No 4, December 2000.

Kerpa, O., Weiss, K and Wörn, H. (2003) "Development of a Flexible Tactile Sensor System for a Humanoid Robot", Proceedings of the 2003 IEEE/RSJ, Int. Conference on Intelligent Robots and Systems, October 2003.

Kim, K., Lee, K. R., Lee, D. S., Cho, N., Kim, W. H., Park, K., Park, H., Kim, Y., Park, Y. and Kim, J. (2006) "A siliconbased flexible tactile sensor for ubiquitous robot companion applications", Institute of Physics Publishing Journalof Physics: Conference Series 34 (2006) pp. 399-403

Lee, M.H. (2000) "Tactile Sensing, New Directions, New Challenges“, The International Journal of Robotics Research, Vol. 19, No. 7, July 2000, pp. 636-643, Sage Publications.

Lee, H.-K., Chang, S.-I. and Yoon, E. (2006) "A Capacitive Proximity Sensor in Dual Implementation with Tactile Imaging Capability on a Single-Flexible Platform for Robot Assistant Applications", Proc. of the 19thInternational Conference on Micro Electro Mechanical Systems MEMS2006, Istambul, Turkey, January 2006, pp. 606-609.

Leineweber, M., Pelz, G., Schmidt, M., Kappert, H., and Zimmer, G. (2000) "New tactile sensor chip with silicone rubber cover", Sensors and Actuators, Vol. 84, pp. 236-245, 2000.

Lomas, T., Tuantranont, A. and Bright, V.M. (2004) "Micromachined Piezoresistive Tactile Sensor Array Fabricated by Bulk-etched MUMPs Process“, NSTI Nanotechnology Conference, 2004.

Mei, T., Li, W. J., Ge, Y., Chen, Y., Ni, L., Chan, M. H. (2000) “An integrated MEMS threedimensional tactile sensor with large force range", Sensors and Actuators, Vol. 80, pp. 155162, 2000.

Ochoteco, E., Pomposo, J.A., Sikora, T., Vidal, F., Martinez, F., Obieta, G., Grande, H. (2008) "All-plastic distributed pressure sensors: taylor-made performance by electroactive materials design”, Microsystem Technologies, Vol 14(8), 1089-1097 (2008). 
Paschen, U., Leineweber, M., Arnelung, J. Schmidt, M. and Zammer, G. (1998) “A Novel Tactile Sensor for Heavy-Load Applications Based on an Integrated Capacitive Pressure Sensor", Sensors and Actuators, Vol. 68, pp. 294-298, 1998.

Salo, T., Vancura, T., Brand, O., and Baltes, H. (2003) "CMOS-Based Sealed Membranes for Medical Tactile Sensor Arrays”, IEEE MEMS, pp. 590-593, Kyoto, Japan, January 19. -23, 2003.

Shan, J. H., Mei, T., Sun, L., Kong, D.Y., Zhang, Z.Y., Ni, L., Meng, M., and Chu, J.R. (2005) "The design and fabrication of a flexible three-dimensional force sensor skin", Proceedings of the IEEE/RSJ International Conference on Intelligent Robots and Systems, 2005 (IROS 2005), pp. 1818-1823.

Shimojo, M., Namiki, A., Ishikawa, M., Makino, R. and Mabuchi, K. (2004) “A Tactile Sensor Sheet Using Pressure Conductive Rubber With Electrical-Wires Stitched Method", IEEE Sensors Journal, Vol. 4, No. 5, October 2004, pp. 589-596

Someya, T., Sekitani, T., Iba, S., Kato, Y., Kawaguchi, H., Sakurai, T., (2004) "A large-area, flexible pressure sensor matrix with organic field-effect transistors for artificial skin applications", Proc Natl Acad 518 Sci USA 101(27):9966-9970, 2004.

Tegin, J. and Wikander, J. (2005) "Tactile sensing in intelligent robotic manipulation - a review", Industrial Robot, Vol. 32, No. 1, pp. 64-70, 2005.

Weiss, K. and Wörn, H. (2004) "Tactile Sensor System for an Anthropomorphic Robotic Hand", IEEE International Conference on Manipulation and Grasping. IMG 2004, Genua, Italy

Wisitsoraat, A., Patthanasetakul, V., Lomas, T., Tuantranont, A. (2007) "Low cost thin film based piezoresistive MEMS tactile sensor”, Sensors and Actuators A 139 (2007) 17-22 